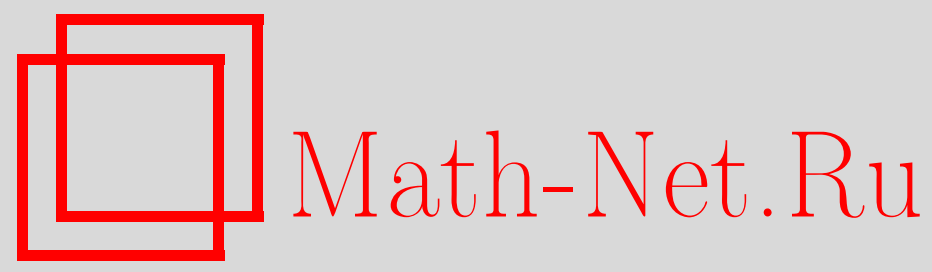

М. Миньотт, Новое доказательство теоремы Ко Чао, Мaтем. заметки, 2004, том 76, выпуск 3, 384-395

DOI: https://doi.org/10.4213/mzm115

Использование Общероссийского математического портала Math-Net.Ru подразумевает, что вы прочитали и согласны с пользовательским соглашением http://www.mathnet.ru/rus/agreement

Параметры загрузки:

IP : 54.197 .217 .227

26 апреля 2023 г., 17:03:45

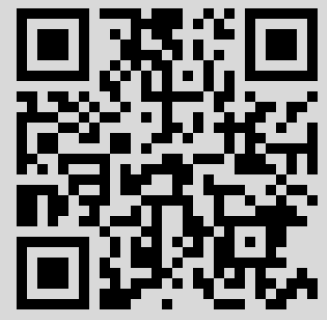


УДК 511.3

\section{НОВОЕ ДОКАЗАТЕЛЬСТВО ТЕОРЕМЫ КО ЧАО}

\section{М. Миньотт}

В статье приводится новое доказательство известной теоремы Ко Чао.

Библиографоия: 13 названий.

Уравнение $x^{p}-y^{2}=1$ было решено В.А. Лебегом [1] еще в 1850 г., но уравнение $x^{p}-y^{2}=-1$ было решено Ко Чао [2] только в 1965 г. Его доказательство было элементарным, однако чрезвычайно изобретательньм (см. [3]). Другое элементарное доказательство было дано Чейном [4], но оно также оказалось достаточно хитроумным (см. [5]). Наша цель - получить доказательство, которое было бы в некотором смысле наиболее естественњым, даже с учетом того, что в нем используется метод Бейкера. Кроме того, наша работа по существу является независимой.

Мы приводим небольшую часть результатов, полученных Нагелем в работах [6] (1921 г.) и [7] (1934 г.), а также адаптируем результат Куммера для первого случая теоремы Ферма. В заключение, мы получаем оценку сверху для показателя $p$, используя линейные формы от двух логарифмов.

1. Две работы Нагеля. Сначала напомним результат, полученньй Эйлером [8] в 1738 г.: уравнение $x^{2}=y^{3}+1$ допускает только иелые решения $(x, y)=( \pm 1,0)$, $(0,-1) u( \pm 3,2)$.

Наша цель - доказать теорему Ко Чао [2] для случая $p \neq 3$.

Теорема 1 (Ко Чао, 1965). Пусть р - нечетное простое число. Тогда уравнение

$$
x^{p}-y^{2}=-1
$$

имеет единственное челое решение $(x, y, p)=(2,3,3)$ с $x y \neq 0$.

Начнем с результатов, представленных в работе [6].

Предположим, что для некоторого нечетного простого числа существуют рациональные целые числа $x$ и $y$ такие, что

$$
x^{p}-y^{2}=-1, \quad x>0, \quad y>0
$$

Если число $y$ четное, то $\operatorname{gcd}(y-1, y+1)=1$ и мы получаем факторизацию $y-1=y_{1}^{p}$, $y+1=y_{2}^{p}$, где $x=y_{1} y_{2}$. Таким образом, выполнено $y_{2}^{p}-y_{1}^{p}=2$, что, очевидно, невозможно при $x>0$. 
Итак, число $y$ нечетное, число $x$ четное, $\operatorname{gcd}(x-1, x+1)=2$, и найдутся $\eta= \pm 1$ и ненулевые рациональные целые числа $u$ и $v$, для которых выполнено

$$
y+\eta=2^{p-1} u^{p}, \quad y-\eta=2 v^{p}, \quad \text { где } x=2 u v \text { и } \operatorname{gcd}(2 u, v)=1 .
$$

Следовательно,

$$
2^{p-2} u^{p}-v^{p}=\eta= \pm 1 .
$$

Чтобы доказать, что $p$ делит $y$, Нагель [6] воспользовался следующей теоремой Штормера (см. [9] или [10], а также [5]).

Теорема 2 (Штормер, 1897). Пусть D - положительное иелое число, не являющееся квадратом, и пусть $\left(u_{1}, v_{1}\right)$ - фундаментальное решение уравнения Пелля-Ферма

$$
u^{2}-D v^{2}= \pm 1
$$

если оно существует. Тогда для любого другого решения $(u, v)$ с $v>v_{1}$ существует простой делитель числа $v$, не делящий число D.

В нашем случае, если $p$ не делит число $y$, то $x+1=x_{1}^{2}$ (в силу леммы 1 , доказываемой ниже, числа $x+1$ и $\left(x^{p}+1\right) /(x+1)$ взаимно просты) и выполняется соотношение

$$
y^{2}-x^{p}=y^{2}-\left(x_{1}^{2}-1\right)\left(\left(x_{1}^{2}-1\right)^{(p-1) / 2}\right)^{2}=1,
$$

так что пара $\left(x_{1},\left(x_{1}^{2}-1\right)^{(p-1) / 2}\right)$ является решением уравнения Пелля-Ферма $u^{2}-$ $\left(x_{1}^{2}-1\right) v^{2}=1$, удовлетворяющим условию $\left(u_{1}, v_{1}\right)=\left(x_{1}, 1\right)$, а это противоречит теореме Штормера. Таким образом (снова в соответствии с леммой 1 ), заключаем, что

$$
x+1=p w^{2}, \quad \frac{x^{p}+1}{x+1}=p z^{2}, \quad \text { где } y=p w z \text { и } \operatorname{gcd}(2 w, z)=1 .
$$

Нам также понадобится другой результат Нагеля [7].

ТЕорема 3 (Нагель, 1921-1934). Если для простого числа $p>3$ диофантово уравнение $x^{p}-y^{2}=-1$ имеет решение $(x, y)$ c $x, y>0$, то $x$ - четное число, $p$ делит у и $\equiv 1(\bmod 8)$.

ДокАЗАТЕЛЬСтво. Мы уже доказали свойства делимости. Остается проверить, что $p \equiv 1(\bmod 8)$. Оригинальное доказательство Нагеля [7] столь элегантно и кратко, что мы полностью его воспроизводим.

Предположим сначала, что $p=8 k+a$, где $a=5,7$. Тогда $x=p w^{2}-1 \equiv a-1(\bmod 8)$. Из (1) следует, что

$$
y^{2}=x^{p}+1=\left(x^{2}-1+1\right)^{4 k} x^{a}+1 \equiv x^{a}+1\left(\bmod x^{2}-1\right) .
$$

Поэтому символ Лежандра равен

$$
\left(\frac{x^{a}+1}{x-1}\right)=\left(\frac{2}{x-1}\right)=+1
$$


что противоречит сравнению $x-1 \equiv 3,5(\bmod 8)$. Следовательно, простое $p$ должно быть сравнимо с $1,3(\bmod 8)$.

Теперь предположим, что $p=8 n+3=24 m+a$, где $a \in\{11,19\}$ (напомним, что $p \neq 3)$. Тогда $x \equiv 2(\bmod 8)$ и

$$
y^{2}=x^{p}+1=\left(x^{3}-1+1\right)^{8 m} x^{a}+1 \equiv x^{a}+1\left(\bmod x^{3}-1\right) .
$$

Таким образом,

$$
\left(\frac{x^{a}+1}{x^{3}-1}\right)=+1
$$

Для случая $a=11$ ввиду $x^{11}-x^{2}=x^{2}\left(x^{9}-1\right)$ и $x+1 \equiv 3(\bmod 8)$ получаем

$$
+1=\left(\frac{x^{2}+1}{x^{3}-1}\right)=\left(\frac{x^{3}-1}{x^{2}+1}\right)=\left(\frac{-x-1}{x^{2}+1}\right)=\left(\frac{x^{2}+1}{x+1}\right)=\left(\frac{2}{x+1}\right)=-1 ;
$$

противоречие. Если же $a=19$, то

$$
+1=\left(\frac{x^{19}+1}{x^{3}-1}\right)=\left(\frac{x+1}{x^{3}-1}\right)=-\left(\frac{x^{3}-1}{x+1}\right)=\left(\frac{2}{x+1}\right)=-1,
$$

и мы снова приходим к противоречию. Теорема 3 доказана.

ЛЕмма 1. Пусть $p$ - нечетное простое число, а с-рачиональное челое число такое, что $|c-1|>1$. Тогда

$$
\operatorname{gcd}\left(\frac{c^{p}-1}{c-1}, c-1\right)= \begin{cases}1, & \text { если р не делит } c-1 \\ p, & \text { в противном случае. }\end{cases}
$$

Кроме того, число $\left(c^{p}-1\right) /(c-1)$ всегда нечетное, а в случае, когда указанныи наибольший общий делитель равен $р$, выполняется сравнение

$$
\frac{c^{p}-1}{c-1} \equiv p\left(\bmod p^{2}\right) \text {. }
$$

2. Частичное исследование уравнения $2^{p-2} u^{p}-v^{p}= \pm 1$. Снова рассмотрим диофантово уравнение, упоминавшееся в п. 1:

$$
2^{p-2} u^{p}-v^{p}=\eta= \pm 1, \quad \text { где } u>0, v>0 \text { и } \operatorname{gcd}(2 u, v)=1 .
$$

В силу (2) имеем $y+\eta=2^{p-1} u^{p}, y-\eta=2 v^{p}$, где $p$ делит $y$. Таким образом, $p \nmid u v$. Применяя еше раз лемму 1 , из соотношения $v^{p}+\eta=2^{p-2} u^{p}$ получаем

$$
\frac{v^{p}+\eta}{v+\eta}=z^{p}
$$

для некоторого положительного рационального целого числа $z$. Полагая $x=-\eta v$, мы можем заменить это уравнение на уравнение

$$
\frac{x^{p}-1}{x-1}=z^{p}
$$

Отметим, что $p \nmid x$, так как $p \nmid v$. Однако это свойство понадобится нам позднее.

Сначала докажем элементарную лемму для уравнения (4). 
ЛЕмма 2. Пусть $x \in \mathbb{Z}$ является решением диофантова уравнения

$$
\frac{x^{p}-1}{x-1}=z^{p}
$$

Тогда $p \nmid z$ и выполняются следующие условия:

(i) $\operatorname{ecлu~} p \mid x$, mo $p^{2} \mid x$;

(ii) $\operatorname{ecлup~} \mid(x+1)$, mo $p^{2} \mid(x+1)$.

ДокАЗАТЕЛЬСтво. Если $p \mid z$, то $p$ делит $x-1$, и по лемме 1 левая часть в (4) сравнима с $p$ по модулю $p^{2}$; противоречие. Таким образом, $p \nmid z$.

Если $p$ делит $x$, то из соотношения

$$
z^{p}=\frac{x^{p}-1}{x-1}=x^{p-1}+\cdots+x+1
$$

следует, что $z \equiv 1(\bmod p)$. Таким образом, $z^{p} \equiv 1\left(\bmod p^{2}\right)$ и $p^{2} \mid\left(x+x^{2}\right)$. Отсюда легко следует утверждение (i).

Теперь предположим, что $p$ делит $x+1$. Положим $x=T-1$; тогда $p \mid T$. Имеем

$$
z^{p}=\frac{x^{p}-1}{x-1}=(T-1)^{p-1}+\cdots+(T-1)+1=1+a T+b T^{2}, \quad a, b \in \mathbb{Z},
$$

где

$$
\begin{aligned}
a & =1-2+3-4+\cdots+(p-2)-(p-1)=(1-2)+(3-4)+\cdots+((p-2)-(p-1)) \\
& =(-1)+(-1)+\cdots+(-1)=-\frac{p-1}{2} .
\end{aligned}
$$

Тогда $z \equiv 1\left(\bmod p^{2}\right)$ и $p^{2} \mid\left(a T+b T^{2}\right)$. Из того, что $p$ делит $T$, но не делит $a$, следует утверждение (ii).

Теперь мы адаптируем доказательство Куммера для "регулярных" простых чисел в первом случае теоремы Ферма. Мы будем следовать изложению Вашингтона [11, гл. I].

Положим $\zeta=\zeta_{p}=e^{2 i \pi / p}$. Будем работать в круговом поле $C=C_{p}=\mathbb{Q}(\zeta)$, для которого кольцом целых чисел является $\mathbb{Z}[\zeta]$. Напомним, что $(1-\zeta)$ является простым идеалом поля $C$ и удовлетворяет соотношению $(1-\zeta)^{p-1}=(p)$. Отметим также соотношения между идеалами $\left(1-\zeta^{j}\right)=(1-\zeta)$ при $j=1,2, \ldots, p-1$ (cp. с $[11$, лемма 1.4]).

Представляя уравнение (4) в виде произведения

$$
\prod_{j=1}^{p-1}\left(x-\zeta^{j}\right)=z^{p}
$$

рассмотрим наибольший общий делитель множителей в левой части. Докажем, что эти множители попарно взаимно просты. Предположим, что простой идеал р поля $C$ делит $\left(x-\zeta^{j}\right)$ и $\left(x-\zeta^{k}\right)$, где $j \neq k$. Исключая $x$, находим $\mathfrak{p} \mid\left(\zeta^{j}-\zeta^{k}\right)$, откуда $\mathfrak{p}=(1-\zeta)$. Однако по лемме 2 вьполнено $p \nmid z$ и, следовательно, $(1-\zeta) \nmid\left(x-\zeta^{j}\right)$, что доказьвает наше утверждение. 
Следовательно, существуют идеалы $\mathfrak{a}_{j}$ кольца $\mathbb{Z}[\zeta]$ такие, что $\left(x-\zeta^{j}\right)=\mathfrak{a}_{j}^{p}$ при $1 \leqslant j<p$. В частности,

$$
(x-\zeta)=\mathfrak{a}^{p} .
$$

Далее будем предполагать, что $p$ является регулярным простым числом, т.е. $p$ не делит число классов поля $C_{p}$. Равенство (5) означает существование алгебраического целого числа $\alpha$, для которого $x-\zeta=$ единица $\cdot \alpha^{p}$. Однако теорема Куммера $[11$, предложение 1.5] утверждает, что для некоторого целого числа $r$ каждая единица поля $C_{p}$ имеет вид $\zeta^{-r} \varepsilon$, где $r \in \mathbb{Z}$ и $\varepsilon$ - действительная единица поля $C$. Таким образом, мы можем записать

$$
x-\zeta=\zeta^{r} \varepsilon \alpha^{p}, \quad r \in \mathbb{Z}, \varepsilon-\text { действительная единица. }
$$

Применяя комплексное сопряжение, находим

$$
x-\zeta^{-1}=\zeta^{-r} \varepsilon \bar{\alpha}^{p} .
$$

Из этих двух соотношений следует, что

$$
x-\zeta-\zeta^{2 r} x+\zeta^{2 r-1}=\zeta^{r} \varepsilon\left(\alpha^{p}-\bar{\alpha}^{p}\right) .
$$

Однако из разложения $\alpha=b_{0}+b_{1} \zeta+\cdots+b_{p-2} \zeta^{p-2}$ с целыми $b_{0}, b_{1}, \ldots, b_{p-2} \in \mathbb{Z}$ имеем $\alpha^{p}-\bar{\alpha}^{p} \equiv\left(b_{0}+b_{1} \zeta^{p}+\cdots+b_{p-2} \zeta^{p(p-2)}\right)-\left(b_{0}+b_{1} \zeta^{-p}+\cdots+b_{p-2} \zeta^{-p(p-2)}\right) \equiv 0(\bmod p)$, откуда получаем соотношение

$$
x-\zeta-\zeta^{2 r} x+\zeta^{2 r-1} \equiv 0(\bmod p)
$$

Так как $\zeta$ имеет степень $p-1$, каждое собственное подмножество множества $\left\{1, \zeta, \zeta^{2}\right.$, $\left.\ldots, \zeta^{p-1}\right\}$ состоит из линейно независимых над $\mathbb{Z}$ компонент. Таким образом, если все числа $1, \zeta, \zeta^{2 r}, \zeta^{2 r-1}$ различны, то мы приходим к противоречию: уравнение (4) не имеет решения.

Если не все числа $1, \zeta, \zeta^{2 r}, \zeta^{2 r-1}$ различны, то возможны следуюшие три случая.

$(0) \zeta^{2 r}=1$. Тогда $(6)$ принимает вид $-\zeta+\zeta^{-1} \equiv 0(\bmod p)$, что невозможно, так как простое число $p$ не делит идеал $(1-\zeta)$.

(1) $\zeta^{2 r-1}=1$. В этом случае $(6)$ влечет $(x+1)(1-\zeta) \equiv 0(\bmod p)$, откуда $x \equiv-1$ $(\bmod p)$. По лемме 2 имеем $p^{2} \mid(x+1)$.

(2) $\zeta^{2 r-1}=\zeta$. Тогда сравнение $(6)$ дает $x\left(1-\zeta^{2}\right) \equiv 0(\bmod p)$, откуда $x \equiv 0(\bmod p)$. Согласно лемме 2 имеем $p^{2} \mid x$.

Подытожим полученное в следующем утверждении.

ТЕОРема 4. Пусть $p$ - регулярное нечетное простое число. Если диофантово уравнение

$$
\frac{x^{p}-1}{x-1}=z^{p}
$$

имеет иелое решение $(x, z)$, то $p \nmid z и p^{2} \mid x(x+1)$.

Возвращаясь к уравнению (3), в котором используются обозначения для $x$ и $y$ из (1), согласно теореме 4 для регулярного простого числа $p$ получаем следующее: либо $p \mid v$, что невозможно, так как $p \mid y$ и $p$ не делит $x=2 u v$, либо $v \equiv \eta(\bmod p)$, откуда $y \equiv 3 \eta(\bmod p)$ и $p=3$, поскольку $p$ делит $y$. Таким образом, имеет место следующий результат. 
ТеОрема 5. Пусть $p>3$ - регулярное простое число. Тогда диофантовы уравнения

$$
2^{p-2} u^{p}-v^{p}=\eta= \pm 1, \quad u>0, \quad v>0
$$

$u$

$$
x^{p}-y^{2}=-1, \quad x>0, \quad y>0 .
$$

не имеют решений.

Из таблицы, приведенной в конще книги [11], находим множество нерегулярных простых чисел:

$$
\mathscr{I}=\{37,59,67,101,103,131,149,157,233,257, \ldots\} .
$$

Замечательньм является то обстоятельство, что числа, которые сравнимы с $1(\bmod 8)$, начинаются с $p=233,257, \ldots$.

Для завершения доказательства теоремы 1 мы воспользуемся следующим результатом, которьй доказывается в приложении.

ТЕОрема 6. Пусть р-нечетное простое число, для которого диофантово уравнение

$$
2^{p-2} u^{p}-v^{p}=\eta= \pm 1, \quad u>0, \quad v>0,
$$

имеет решение. Тогда $p<233$.

ЗАмЕчАниЕ 1. Вместо критерия Нагеля, полученного в 1934 г., мы можем попытаться применить критерий, полученньй им же в 1921 г. (доказательство последнего основано на использовании периодов Гаусса и квадратичных полей), для которого множество исключительньх простых $p$ имеет вид

$$
\{31,59,73,83,89,107,113,139,211,223,233,239,251,257, \ldots\} \text {. }
$$

Теперь его пересечение с множеством $\mathscr{I}$ имеет вид $\{59,223,257, \ldots\}$, и проблема возникает при $p=59$.

ЗАмЕчаниЕ 2. Используя гипергеометрические функции, М. Беннетт смог получить оценку сверху $p<83$ вместо нашей оценки $p<233$; однако такая хорошая оценка нам здесь не понадобилась.

ЗАмЕчАниЕ 3. В литературе некоторые авторы также используют следующий критерий: если уравнение $2^{p-2} u^{p}-v^{p}=\eta= \pm 1$ имеет нетривиальное решение для простого числа $p>3$, то $2^{p-1} \equiv 1\left(\bmod p^{2}\right)$ и $3^{p-1} \equiv 1\left(\bmod p^{2}\right)$. Например, в работе [12] используется тот факт, что объединение этих сравнений дает неравенство $p>100000$. Однако нам не хотелось бы применять этот результат ввиду недоступности и непонятности аргументов оригинальной статьи [12]. Во всяком случае подобньй результат оказывается менее элементарным, чем критерий, которым воспользовались мы.

ЗАмечАниЕ 4. Конечно, теорема Ко Чао также является тривиальньм следствием подходящего обобщения теоремы Уайлса о неразрешимости уравнения Ферма. Но опять-таки подобную аргументацию нельзя отнести к элементарной. 
Приложение. Оценка сверху для $p$. Рассмотрим диофантово уравнение из п. 1 :

$$
2^{p-2} u^{p}-v^{p}= \pm 1, \quad u, v>0 .
$$

Нетрудно получить оценку снизу

$$
v \geqslant 2^{p^{2}-2 p-1} .
$$

Рассмотрим линейную форму

$$
\Lambda=p \log \frac{2 u}{v}-2 \log 2=\log \left(1 \pm \frac{1}{v^{p}}\right) .
$$

Достаточно показать, что

$$
0<|\Lambda|<\frac{2}{v^{p}},
$$

откуда немедленно следует, что

$$
\log |\Lambda|<-p \log v+1 .
$$

Чтобы получить хорошую оценку сверху для $p$, мы воспользуемся главным результатом работы [13]. Напомним его, чтобы помочь читателю проследить за нашими вычислениями.

Теорема А. Пусть $\alpha_{1}$ и $\alpha_{2}-$ два не равных нулю алгебраических числа таких, что $\left|\alpha_{1}\right| \geqslant 1 u\left|\alpha_{2}\right| \geqslant 1$. Пусть $\log \alpha_{1} u \log \alpha_{2}-$ их логарифмы по любому основанию. Положим

$$
\Lambda=b_{2} \log \alpha_{2}-b_{1} \log \alpha_{1},
$$

где $b_{1}$ и $b_{2}$ - положительные челые числа, и определим

$$
D=\frac{\left[\mathbb{Q}\left(\alpha_{1}, \alpha_{2}\right): \mathbb{Q}\right]}{\left[\mathbb{R}\left(\alpha_{1}, \alpha_{2}\right): \mathbb{R}\right]} .
$$

Пусть $K \geqslant 2$ - челое число, $L, R_{1}, R_{2}, S_{1}, S_{2}$ - положительные челье числа, пусть $\rho>1$ - действительное число. Предположим, что

$$
R_{1} S_{1} \geqslant L, \quad R_{2} S_{2}>(K-1) L .
$$

Положим $R=R_{1}+R_{2}-1, S=S_{1}+S_{2}-1, g=1 / 4-K L /(12 R S)$, и определим

$$
b=\left((R-1) b_{2}+(S-1) b_{1}\right)\left(\prod_{k=1}^{K-1} k !\right)^{-2 /\left(K^{2}-K\right)} .
$$

Пусть $a_{1}$ и а $a_{2}-$ действительные числа такие, что

$$
(\rho-1) \log \alpha_{i}+2 D h\left(\alpha_{i}\right) \leqslant a_{i}, \quad i=1,2 .
$$

Предположим, что $\alpha_{1}$ и $\alpha_{2}$ мультипликативно независимь, числа $r b_{2}+s b_{1}$, әде $0 \leqslant r \leqslant R-1 u 0 \leqslant s \leqslant S-1$, попарно различны $и$

$$
K(L-1) \log \rho-(D+1) \log (K L)-D(K-1) \log \left(\frac{b}{2}\right)-g L\left(R a_{1}+S a_{2}\right)>0 .
$$


Тогда имеет место оченка снизу

$$
\left|\Lambda^{\prime}\right| \geqslant \rho^{-K L+0.5}
$$

əде

$$
\Lambda^{\prime}=\Lambda \cdot \max \left\{\frac{L S e^{L S|\Lambda| /\left(2 b_{2}\right)}}{2 b_{2}}, \frac{L R e^{L R|\Lambda| /\left(2 b_{1}\right)}}{2 b_{1}}\right\} .
$$

Чтобы получить наиболее точную оценку для $p$, предположим, что

$$
p \geqslant 233 \quad(\text { так что } \log p=5.451038453 \ldots),
$$

и применим теорему А, выбирая

$$
a_{1}=2 \log (e v)\left(1+\frac{2(\rho-1)}{p^{3}}\right) \quad \text { и } \quad a_{2}=(\rho+1) \log 2 .
$$

Выбор $a_{2}$, очевидно, возможен. Обсудим подробнее выбор $a_{1}$. Согласно условиям теоремы А этот параметр должен удовлетворять неравенству

$$
a_{1} \geqslant(\rho-1) \log \alpha_{1}+2 h\left(\alpha_{1}\right) .
$$

Из первой оценки сверху для $|\Lambda|$ получаем $\left|\log \alpha_{1}\right|<(2.001 / p) \log 2$ и, очевидно, вьполнено $h\left(\alpha_{1}\right) \leqslant \log (e v)$, где $v>2^{p^{2}-2 p-1}$. Это обосновывает наш выбор $a_{1}$.

Возьмем $22 \leqslant \rho \leqslant 24$. Имеем $a_{1}>2 \log \left(2^{p^{2}-2 p}\right)$, откуда

$$
a_{1}>2\left(p^{2}-2 p\right) \log 2>74614, \quad a_{2}=(\rho+1) \log p \geqslant 23 \log p>15.9423 .
$$

Предположим, что

$$
5 \leqslant L \leqslant 1.5 \log p .
$$

Возьмем $K=\left[\mu^{2} L a_{1} a_{2}\right]+1$, где $\mu$ - действительное число, которое мы выберем позднее так, чтобы оно удовлетворяло неравенствам

$$
0.2 \leqslant \mu \leqslant 0.4 .
$$

Тогда

$$
K \geqslant\left\lceil 0.2^{2} \cdot 5 \cdot a_{1} a_{2}\right\rceil \geqslant 237906>47000 L
$$

и, чтобы выполнялось условие (9), положим

$$
R_{1}=1, \quad S_{1}=L, \quad R_{2}=\left[\mu L a_{2}\right]+1, \quad S_{2}=\left[\mu L a_{1}\right]+1 .
$$

Если сушествуют два цельх числа $r_{0}$ и $s_{0},\left|r_{0}\right|<R$ и $\left|s_{0}\right|<S$ таких, что $r_{0} b_{2}+s_{0} b_{1}=0$, то $p$ делит $r_{0}$ и, следовательно,

$$
p<R \leqslant 1+1.5 \mu L(\rho+1) \log 2<1+1.5 \cdot 0.4 \cdot 25 \cdot \log p \cdot \log 2,
$$

откуда следует, что $p<40$, а это противоречит предположению о том, что $p \geqslant 233$. Таким образом, числа $r b_{2}+s b_{1}(0 \leqslant r \leqslant R-1,0 \leqslant s \leqslant S-1)$ попарно различны. 
Имеет место следующая общая оценка сверху для $b$ (см. [13, лемма 6$])$ :

$$
b \leqslant \frac{\left((R-1) b_{2}+(S-1) b_{1}\right)}{K-1} \exp \left\{\frac{3}{2}-\frac{\log (2 \pi(K-1) / \sqrt{e})}{K-1}+\frac{\log K}{6 K(K-1)}\right\} .
$$

Сначала рассмотрим величину $(R-1) b_{2}+(S-1) b_{1}$. Напомним, что $R=R_{1}+R_{2}-1$ и $S=S_{1}+S_{2}-1$. Таким образом, используя определение чисел $R_{1}, R_{2}, S_{1}$ и $S_{2}$, получаем

$$
(R-1) b_{2}+(S-1) b_{1} \leqslant b_{1}\left(\mu L a_{1}+L-1\right)+b_{2}\left(\mu L a_{2}\right)<\left(\frac{b_{1}}{a_{2}}+\frac{b_{2}}{a_{1}}\right) \mu L a_{1} a_{2}\left(1+\frac{1}{\mu a_{1}}\right) .
$$

По определению

$$
b^{\prime}=(\rho+1)\left(\frac{b_{1}}{a_{2}}+\frac{b_{2}}{a_{1}}\right)
$$

и предыдущая оценка может быть записана в виде

$$
(R-1) b_{2}+(S-1) b_{1}<\frac{b^{\prime}}{(\rho+1)} \cdot \mu L a_{1} a_{2} \cdot\left(1+\frac{1}{\mu a_{1}}\right) .
$$

Благодаря нашим предположениям относительно $L$ и $K$, отсюда следует, что

$$
\begin{aligned}
\log b & \leqslant 1.5+\log \left(\frac{\mu L a_{1} a_{2}}{(\rho+1)(K-1)}\right)+\log b^{\prime}+\frac{1}{\mu a_{1}}-\frac{\log (3.8 K)}{K-1} \\
& \leqslant 1.5-\log ((\rho+1) \mu)+\log b^{\prime}+\frac{1}{\mu a_{1}}-\frac{\log (3.8 K)}{K-1} .
\end{aligned}
$$

Так как

$$
b^{\prime}=(\rho+1)\left(\frac{b_{1}}{a_{2}}+\frac{b_{2}}{a_{1}}\right)=\frac{(\rho+1) b_{1}}{a_{2}}\left(1+\frac{a_{2} b_{2}}{a_{1} b_{1}}\right) \leqslant \frac{p}{\log 2}\left(1+\frac{(\rho+1)}{p^{2}(p-2)}\right),
$$

окончательно получаем

$$
\log b \leqslant 1.5-\log ((\rho+1) \mu)+\log \left(\frac{p}{\log 2}\right)+\frac{(\rho+1)}{p^{2}(p-2)}+\frac{1}{\mu a_{1}}-\frac{\log (3.8 K)}{K-1} .
$$

Теперь рассмотрим величину $g$ из формулировки теоремы А. Согласно соотношениям

$$
R=R_{1}+R_{2}-1 \leqslant \mu L a_{2}+1, \quad S=S_{1}+S_{2}-1 \leqslant \mu L a_{1}+L
$$

находим (очевидно, $g$ - возрастающая функция от $R$ и $S$ )

$$
\begin{aligned}
g L\left(R a_{1}+S a_{2}\right)= & \frac{1}{4} L\left(R a_{1}+S a_{2}\right)-\frac{K L^{2}}{12}\left(\frac{a_{1}}{S}+\frac{a_{2}}{R}\right) \\
\leqslant & \frac{1}{4} L\left(\mu L a_{1} a_{2}+a_{1}+\mu L a_{1} a_{2}+L a_{2}\right) \\
& -\frac{\mu^{2} L^{3} a_{1} a_{2}+L^{2}}{12}\left(\frac{a_{1}}{\mu L a_{1}+L}+\frac{a_{2}}{L a_{2}+1}\right) .
\end{aligned}
$$


Применяя теперь соотношения (для положительных переменных)

$$
\frac{1}{x+y}=\frac{1}{x}-\frac{y}{x^{2}}+\frac{y^{2}}{(x+y) x^{2}} \geqslant \frac{1}{x}-\frac{y}{x^{2}}
$$

получаем

$$
\begin{aligned}
g L\left(R a_{1}+S a_{2}\right) \leqslant & \frac{\mu L^{2} a_{1} a_{2}}{2}+\frac{L a_{1}}{4}+\frac{L^{2} a_{2}}{4}-\frac{\mu^{2} L^{2} a_{1} a_{2}}{6}+\frac{a_{2} L^{2}}{12} \\
& +\frac{a_{1} L}{12}-\frac{L}{6 \mu}+\frac{L}{12 \mu^{2}}\left(\frac{1}{a_{1}}+\frac{1}{L a_{2}}\right) .
\end{aligned}
$$

В силу оценок для $a_{1}, a_{2}$ и $\mu$ это влечет

$$
g L\left(R a_{1}+S a_{2}\right) \leqslant \frac{\mu L^{2} a_{1} a_{2}}{3}+\frac{L a_{1}}{3}+\frac{L^{2} a_{2}}{3} .
$$

Действительно, так как $\mu \geqslant 0.2$ и $a_{1}>L a_{2}=4(\rho+1) \log 2 \geqslant 92 \log 2$, вьполнено

$$
\frac{L}{6 \mu}>\frac{L}{12 \mu^{2}}\left(\frac{1}{a_{1}}+\frac{1}{L a_{2}}\right)
$$

Применяя эти соображения, видим, что условие (10) выполняется, если

$$
\begin{aligned}
\Phi= & K(L-1) \lambda+(K-1) \log 2-2 \log (K L)-\frac{\mu L^{2} a_{1} a_{2}}{3}-\frac{L a_{1}}{3}-\frac{L^{2} a_{2}}{3} \\
& -(K-1)\left(1.5-\log ((\rho+1) \mu)+\log \left(\frac{p}{\log 2}\right)+\frac{\rho+1}{p^{2}(p-2)}+\frac{L-1}{\mu a_{1}}-\frac{\log (3.8 K / e)}{K-1}\right) \\
> & 0,
\end{aligned}
$$

где положено $\lambda=\log \rho$. Тогда

$$
\Phi \geqslant \Phi_{0}+\Theta
$$

где

$$
\begin{aligned}
\Phi_{0}= & K(L-1) \lambda+K \log \left(\frac{2}{\theta}\right)-(K-1)\left(1.5-\log ((\rho+1) \mu)+\log \left(\frac{p}{\log 2}\right)\right) \\
& -\frac{L^{3 / 2}}{3} \sqrt{(K-1) a_{1} a_{2}}
\end{aligned}
$$

с новьм параметром $\theta>1$, который выбирается ниже, и

$$
\Theta=K \log \theta-\log \left(K L^{2}\right)-\frac{L a_{1}}{3}-\frac{L^{2} a_{2}}{3}-K \frac{\rho+1}{p^{2}(p-2)}-\frac{L^{2} \mu}{a_{2}}-\frac{(L-1) K}{\mu a_{1}}-\log \left(\frac{e}{1.9}\right)
$$


Параметр $\theta$ должен быть выбран так, чтобы $\Theta>0$. Заметим, что в силу (7), (11) и условий на параметры имеем

$$
\begin{gathered}
\frac{\log \left(K L^{2}\right)}{K}<\frac{\log \left(K^{3} / 47000^{2}\right)}{K}<0.000066, \\
\frac{L a_{1}}{3 K}<\frac{1}{3 \mu^{2} a_{2}} \leqslant \frac{1}{0.12(\rho+1) \log 2} \leqslant \frac{1}{0.12 \cdot 23 \log 2}<0.5227156, \\
\frac{L^{2} a_{2}}{3 K}<\frac{L}{3 \mu^{2} a_{1}}<0.0009133, \quad \frac{\rho+1}{p^{2}(p-2)}<0.0000012, \\
\frac{(L-1) K}{\mu a_{1} K}<\frac{L}{0.2 \cdot a_{1}}<0.000548 \quad \text { и } \quad \frac{\log (e / 1.9)}{K}<0.00000151 .
\end{gathered}
$$

Таким образом, достаточно выбрать

$$
\log \theta=0.522716 \text {. }
$$

Тогда, после деления $\Phi_{0}$ на $\mu L a_{1} a_{2}$ видим, что условие (10) выполняется, если

$$
\mu\left((L-1) \lambda+\log \left(\frac{2}{\theta}\right)-1.5+\log (\rho+1)-\log \left(\frac{p}{\log 2}\right)\right)+\mu \log \mu-\frac{L}{3} \geqslant 0 .
$$

В таком случае, в силу (10) и неравенства $R+S \leqslant K$ имеем

$$
\log |\Lambda| \geqslant-K L \log \rho-\log (K L) .
$$

Затем, сравнивая (8) и (12), получаем

$$
p \leqslant 2.00001 \mu^{2} L^{2}(\rho+1) \log 2 \cdot \log \rho .
$$

Так как $\lambda=\log \rho$, условие (12) эквивалентно следующему условию:

$$
L\left(\mu \log \rho-\frac{1}{3}\right) \geqslant \mu\left(\eta-\log \left(1+\frac{1}{\rho}\right)+\log \left(\frac{p}{\log 2}\right)-\log \mu\right),
$$

где положено $\eta=1.5-\log (2 / \theta)$. Выберем

$$
\mu=\frac{2}{3 \log \rho} ;
$$

тогда предыдущее условие принимает вид

$$
L \geqslant 3 \cdot \frac{2}{3 \log \rho} \cdot\left(\eta-\log \left(1+\frac{1}{\rho}\right)+\log \left(\frac{p}{\log 2}\right)-\log \mu\right) .
$$

При $\rho=22.9$ (значит, $\mu=0.21291520802499 \ldots \in[0.2,0.4]$ ) видим, что последнее неравенство вьполняется, если

$$
L \geqslant 0.63875 \cdot(\log p+3.202),
$$

так что мы можем взять

$$
L=[0.63875 \cdot \log p+3.05] .
$$

(Проверим условие $5 \leqslant L \leqslant 1.5 \log p$. Так как $p \geqslant 233$, имеем $6 \leqslant L \leqslant 1.23 \log p$. Это завершает проверку.) Тогда

$$
p \leqslant 2.0001 \cdot \mu^{2}(\rho+1) \log \rho \cdot \log 2 L^{2}<4.7032 L^{2} \leqslant 4.7032(0.63875 \cdot \log p+3.05)^{2},
$$

откуда

$$
p \leqslant 1.919(\log p+4.775)^{2} .
$$

Это неравенство дает оценку сверху $p \leqslant 193$, что противоречит первоначальному предположению о том, что $p \geqslant 233$. Теорема 6 доказана. 


\section{СПИСОК ЦИТИРОВАННОЙ ЛИТЕРАТУРЫ}

[1] Lebesgue V.A. Sur l'impossibilité, en nombres entiers, de l'équation $x^{m}=y^{2}+1 / /$ Nouv. Ann. Math. 1850. V. 9. P. 178-181.

[2] Ko Chao. On the diophantine equation $x^{2}=y^{n}+1, x y \neq 0 / /$ Sci. Sinica. 1965. V. 14. P. 457-460.

[3] Mordell L. J. Diophantine equations. Pure Appl. Math. V. 30. London-New York: Academic Press, 1969.

[4] Chein E.Z. A note on the equation $x^{2}=y^{q}+1 / /$ Proc. Amer. Math. Soc. 1976. V. 56. P. 83-84.

[5] Ribenboim R. Consecutive powers // Expositiones Math. 1984. V. 2. P. 193-221.

[6] Nagell T. Sur l'impossibilité de l'équation indéterminée $z^{p}+1=y^{2} / /$ Norsk Mat. Forenings Skrifter. 1921. V. 1. № 4.10 pages.

[7] Nagell T. Sur une équation à deux indéterminées // Norsk Vid Selsk Forh. 1934. V. 7. P. 136-139.

[8] Euler L. Theorematum quorandam arithmeticorum demonstrationes; // Comm. Acad. Sci. Petropolitanae. 1738. V. 10. P. 125-146.

[9] Størmer C. Quelques théorèmes sur l'équation de Pell $x^{2}-D y^{2}= \pm 1$ et leurs applications // Christiania. Videnskabens Selskabs Skifter, Math. Nat. Kl. 1897. № 2. 48 pages.

[10] Størmer C. Solution d'un problème curieux qu'on rencontre dans la théorie élémentaire des logarithmes // Nyt Tidskrift für Math. (Copenhagen) Ser. B. 1908. V. 19. P. 1-7.

[11] Washington L. C. Introduction to Cyclotomic Fields. New York: Springer-Verlag, 1982.

[12] Inkeri K., Hyyrö S. On the congruences $3^{p-1} \equiv 1\left(\bmod p^{2}\right)$ and the diophantine equation $x^{2}-1=y^{p} / /$ Ann. Univ. Turku Ser. A. 1961. P. 27-30.

[13] Laurent M., Mignotte M., Nesterenko Y. Formes linéaires en deux logarithmes et déterminants d'interpolation // J. Number Theory. 1995. V. 55. P. 285-321. 